\title{
The effectiveness of corticosteroid injection in the treatment of plantar fasciitis
}

Teck Wee Andrew Ang, MBBS, MMED

\begin{abstract}
Plantar fasciitis is a common cause of heel pain in adults. Although it is usually a self-limiting condition, the pain may become prolonged and severe enough to cause significant distress and disruption to the patient's daily activities and work. PubMed and Cochrane Central Register of Controlled Trials databases were searched for randomised controlled trials (RCTs) and a total of ten RCTs were selected for evaluation. These RCTs involved the use of either palpation- or ultrasonography-guided corticosteroid injections in patients diagnosed with plantar fasciitis. All placebo-controlled RCTs showed a significant reduction in pain with the use of corticosteroid injections. Some studies also showed that corticosteroid injections yielded better results than other treatment modalities. However, it is evident from these studies that the effects of corticosteroid injections are usually short-term, lasting 4-12 weeks in duration. Complications such as plantar fascia rupture are uncommon, but physicians need to weigh the treatment benefits against such risks.
\end{abstract}

Keywords: corticosteroid injection, effectiveness, heel pain, plantar fasciitis, randomised controlled trials

\section{INTRODUCTION}

Plantar fasciitis is one of the most common causes of heel pain, accounting for about one million patient visits per year in the United States. ${ }^{(1)}$ Although it is usually a self-limiting condition with a majority of cases resolving within ten months, about $10 \%$ of patients develop chronic plantar fasciitis. ${ }^{(2)}$ Many patients seek help from their family physicians and foot specialists when the pain becomes severe enough to cause significant distress and disruption to their daily activities and work.

Plantar fasciitis is used to describe heel pain caused by an inflammation of the plantar fascia. This could result from a one-off tear in the plantar fascia or damage from repetitive microtraumas. Plantar fasciosis describes the degenerative, non-inflamed phase of plantar fasciopathy. It is an enthesopathy that arises from degenerative processes affecting the junction between the periosteal (calcaneus) and the ligament attachment (plantar fascia). ${ }^{(3)}$

Plantar fasciitis can affect both athletes and sedentary people, particularly middle-aged and older individuals. ${ }^{(4)}$ Intrinsic risk factors include obesity, pes planus, pes cavus and a shortened Achilles tendon. Extrinsic risk factors include walking on hard surfaces or barefoot, prolonged weight bearing, inadequate stretching and poor footwear. ${ }^{(5)}$ People who walk more during work are shown to be at a higher risk for developing this condition. ${ }^{(6)}$

Although there are many treatment modalities for plantar fasciitis, there is little consensus on its clinical approach. To date, there is no single treatment supported by the highest level of evidence. High-quality studies involving double-blinded, placebo-controlled randomised controlled trials (RCTs) are hard to come by due to the debilitating pain experienced by most patients during the initial consultation. Another possible reason is the fact that most therapies are used in combination ${ }^{(7)}$ and thus there is poor evidence on which modality is the best.
A systematic review of treatments for painful heels conducted by Atkins et $\mathrm{al}^{(8)}$ in 1999 found that although much has been written about the treatment of plantar heel pain, the number of RCTs in the literature was small and most cases involved small populations of patients, which limited the generalisability of treatment efficacy.

Corticosteroid injections have been used to treat plantar heel pain since the 1950s. ${ }^{(9)}$ Both orthopaedic surgeons and rheumatologists have been known to use them frequently. ${ }^{(10)}$ The advantages of corticosteroid injections include low cost, low complexity and rapid pain relief (i.e. it can be administered by most family physicians in an outpatient setting). However, many are concerned about the potential complications associated with this treatment modality, which may offset its benefits. Thus, the recommendation of corticosteroid injections as an initial or tier 1 treatment option by the American College of Foot and Ankle Surgeons (ACFAS) ${ }^{(11)}$ was met with much scepticism and raised certain controversial issues. To further complicate matters, in recent years, the advent of other injectable options (e.g. plateletrich plasma, autologous blood and botulinum toxin) have also made it more difficult for family physicians to decide on the most appropriate course of action for their patients.

Many studies have been done to evaluate the efficacy of corticosteroid injections for the treatment of plantar fasciitis. Most compare its efficacy with that of other treatment modalities. However, these modalities contain inherent differences, even within the corticosteroid injection arm, such as the method of injection, type of steroid used, concurrent use of local anaesthetic and physical therapy, and use of ultrasonography (US) guidance and nerve blocks.

This review aims to examine the current evidence available and to provide evidence-based recommendations for family physicians on the use of corticosteroid injections in patients suffering from plantar fasciitis. 


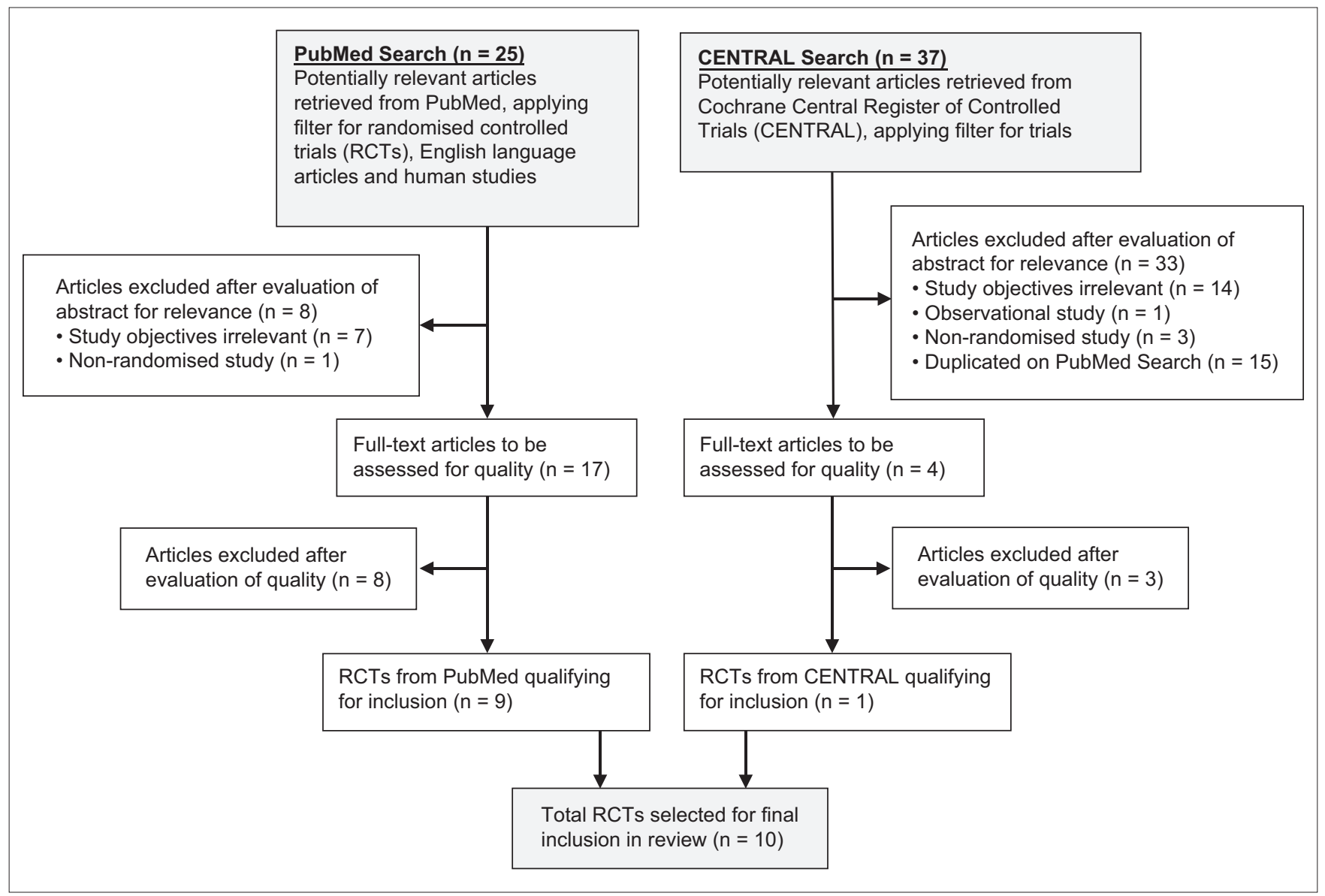

Fig. 1 Flow chart shows the study selection process.

\section{METHODOLOGY}

\section{Data sources}

A data search was performed on PubMed and the Cochrane Central Register of Controlled Trials (CENTRAL) databases up to 10 August 2014. The following search strategy was used: [plantar fasciitis OR heel pain OR painful heel OR plantar fasciosis OR plantar fasciopathy] AND [corticosteroid injection OR steroid injection OR glucocorticoid injection].

\section{Study selection}

RCTs that studied the use of corticosteroid injections in patients with plantar fasciitis and had Jadad scores $\geq 3$ were included. After filtering for RCTs, human studies and English-language articles, a PubMed search yielded 25 potentially relevant articles. Of the 25 studies, seven did not have objectives that were relevant to this review and one was a non-randomised study. Upon reviewing the full-text articles of the remaining 17 studies, six had Jadad scores $<3$, one was a not a RCT and one had no trial results. The remaining nine studies were selected for review.

A similar search performed on CENTRAL yielded 37 potentially relevant articles after filtering for trials. Of these, 15 were duplicated on the PubMed search, 14 had objectives not relevant to this review and four were not RCTs. Upon review of the four remaining full text articles, three were excluded due to Jadad scores $<3$, leaving only one study for inclusion in this review. Thus, a total of ten RCTs were selected for review. This selection process is depicted in Fig. 1.

\section{Data extraction}

The following data was extracted from each included study: study design; Jadad score; study population; duration of heel pain; prior treatment; type, amount and method of corticosteroids injections; use of local anaesthetic; use of nerve blocks; outcome measures; results; adverse events; and dropout numbers. These results are summarised in Table I.

\section{RESULTS}

\section{Study quality}

The Jadad score was used to measure the likelihood of bias and thus the quality of the selected RCTs. ${ }^{(12)}$ Two of the RCTs had a Jadad score of $5,{ }^{(13,14)}$ two had a score of $4^{(7,15)}$ and six had a score of $3 .^{(16-21)}$

\section{Characteristics of the study population}

The mean age of the study populations of the ten included RCTs was 41.4-57.0 years. The duration of their symptoms was 2-180 months, with the majority suffering from plantar heel pain for at least six months. ${ }^{(7,13,16,17,19-21)}$ Five of the ten RCTs included study populations that had failed conservative therapies for at least 2-6 months. ${ }^{(13,15,19,21)}$ 


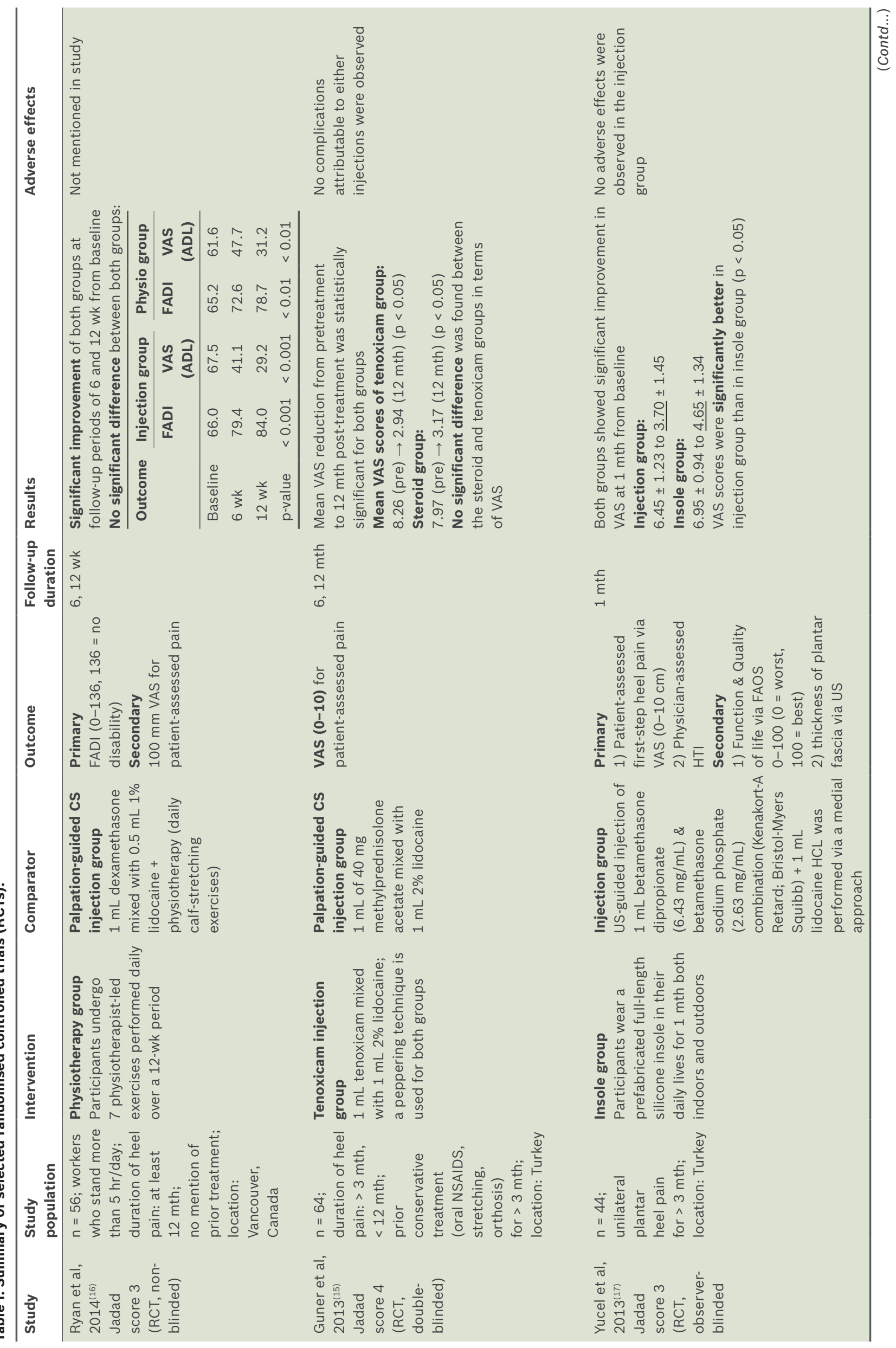




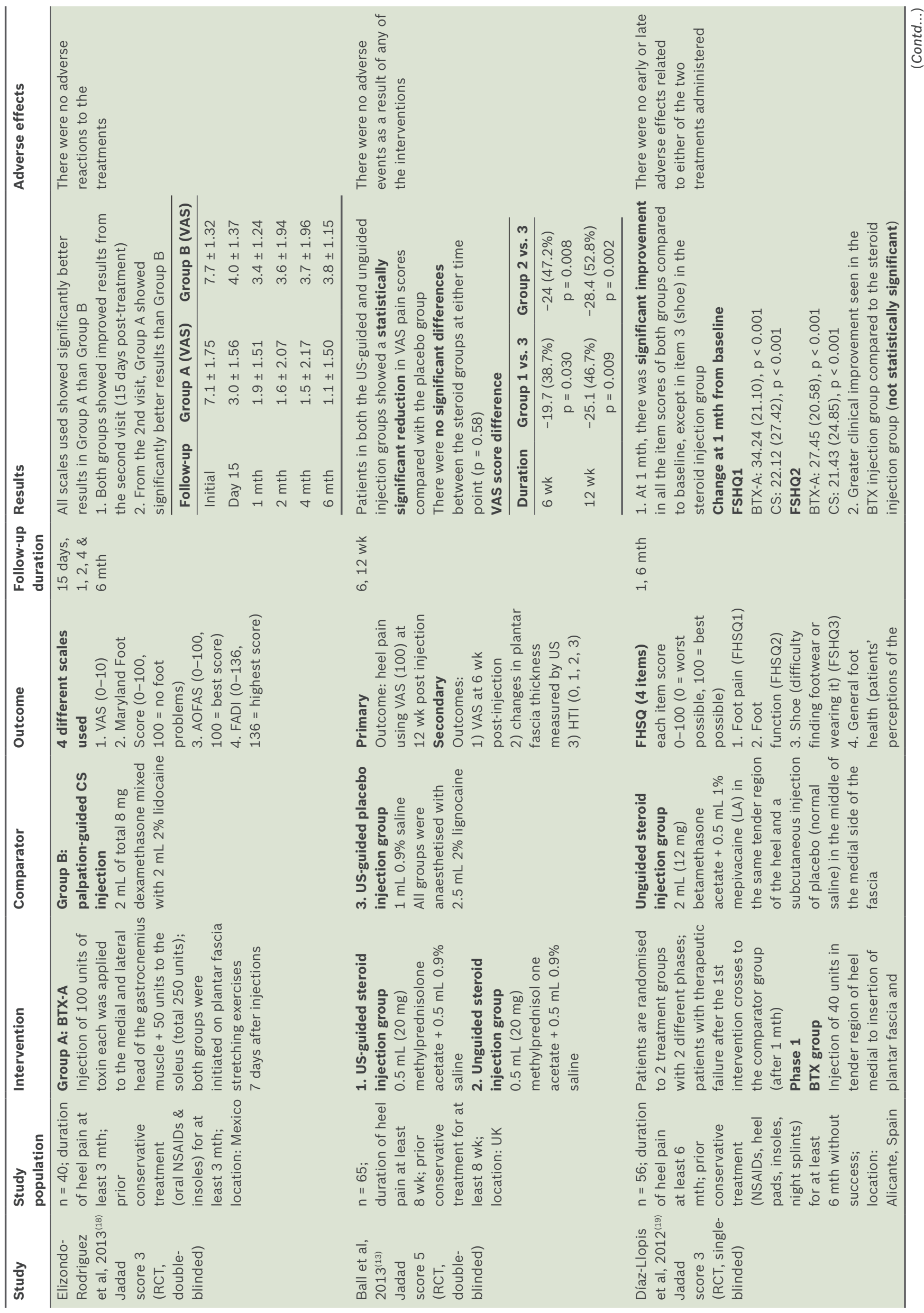




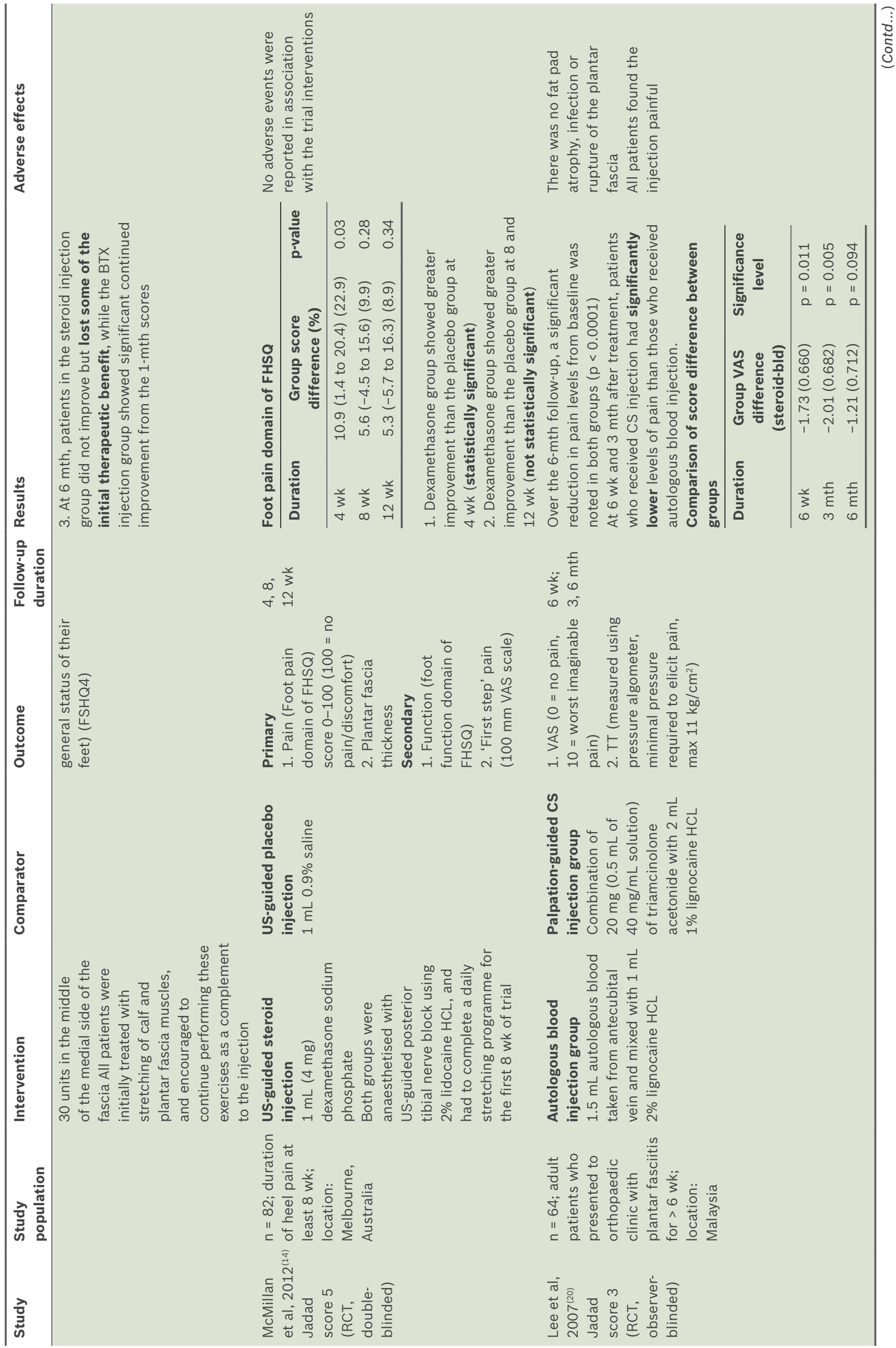









\section{Corticosteroid injections}

Different corticosteroids were used for the injections in the studies. Five RCTs explored the use of long-acting corticosteroids, i.e. dexamethasone ${ }^{(14,16,18)}$ and betamethasone, ${ }^{(17,19)}$ while the other five investigated the use of intermediate-acting corticosteroids, i.e. methylprednisolone, ${ }^{(13,15,21)}$ prednisolone $^{(7)}$ and triamcinolone. ${ }^{(20)}$ To guide the corticosteroid injections, seven RCTs used the palpation method, ${ }^{(7,15,16,18-21)}$ two used USguidance, ${ }^{(14,17)}$ and one used both US- and palpation-guided injections in different arms. ${ }^{(13)}$ Three approaches of injections were employed in the studies: eight RCTs adopted the medial approach, ${ }^{(7,14-20)}$ one adopted the posterior approach ${ }^{(13)}$ and one involved injections through the plantar aspect of the heel pad. ${ }^{(21)}$

\section{Outcomes}

The main outcomes of the studies reviewed fall into the three following categories: (a) patient-assessed outcomes; (b) physicianassessed outcomes; and (c) disease-oriented outcomes. The results for category (a) are summarised in Table I, while those for categories (b) and (c) are summarised in Table II.

\section{Patient-assessed outcome: foot pain}

The measurement of foot or heel pain is one of the main outcomes. The instruments used to measure foot pain include the Visual Analogue Scale (VAS) and the foot pain domain of the Foot Health Status Questionnaire (FHSQ). All studies used the VAS as one of the scales to measure foot pain, except McMillan et al and Díaz-Llopis et al, which used the FHSQ. ${ }^{(14,19)}$

Two placebo-controlled RCTs ${ }^{(13,14)}$ reported significantly reduced pain scores within the corticosteroid injection groups compared to the placebo groups. The study by Ball et al showed up to $47.2 \%$ and $52.8 \%$ pain reduction at six and 12 weeks, respectively, in the corticosteroid injection arm compared to the placebo arm. McMillan et al reported an improvement of foot pain scores in the corticosteroid injection arm compared to the placebo arm at the four-, eight- and 12-week follow-up. However, the difference in foot pain scores was only significant at the four-week mark, with a $22.9 \%$ pain reduction in the intervention group.

Three studies showed significant pain reduction in the corticosteroid injection group compared to the other types of intervention, namely use of insole, ${ }^{(17)}$ autologous blood injection $^{(20)}$ and local anaesthetic injection with or without tibial nerve block. ${ }^{(7)}$ One study ${ }^{(18)}$ reported better results in the botulinum toxin A injection group (intervention group) compared to the corticosteroid injection group. The remaining four studies ${ }^{(15,16,19,21)}$ showed significant pain reduction in both intervention groups at follow-up intervals when compared to baseline but no significant differences between the intervention groups.

A variety of scales were used to measure other outcomes such as foot function, foot health and quality of life. Some of these scales were not designed to assess patients with plantar fasciitis; for example, the Maryland Foot Score was designed to assess foot injuries, the American Orthopaedic Foot and Ankle Society's Ankle-Hindfoot Scale was designed to assess ankle and hindfoot joint injuries, while the Foot and Ankle Disability Index
(FADI) is used to detect functional limitations in subjects with chronic ankle instability. However, all three scales were used in conjunction with VAS in the studies ${ }^{(16,18,21)}$ concerned.

\section{Physician-assessed outcomes}

The two physician-assessed outcomes used in the studies were the Heel Tenderness Index (HTI) and Tenderness Threshold (TT). Ball et $\mathrm{al}^{(13)}$ showed that HTI improved significantly in the steroid injection groups compared to the placebo group at the 12-week follow-up. Yucel et al ${ }^{(17)}$ found significant improvement in HTI in both the US-guided/steroid injection group and the insole group from baseline, although there was no significant difference between the two groups. To measure TT, Lee et $\mathrm{al}^{(20)}$ used a pressure algometer, in which the minimal pressure required to elicit pain was defined as the TT recorded on the 11-kg range algometer (i.e. maximal pressure is $11 \mathrm{~kg} / \mathrm{cm}^{2}$ ). Lee et al's study found that the steroid group had a significantly higher TT than the autologous blood group at the six-week, three-month and six-month post-treatment follow-up. ${ }^{(20)}$

\section{Disease-oriented outcomes}

Three studies ${ }^{(13,14,17)}$ measured plantar fascia thickness as one of the outcomes. Both the placebo-controlled trials ${ }^{(13,14)}$ showed that the steroid group had a significantly greater reduction in plantar fascia thickness than the placebo group at each follow-up interval. Yucel et $\mathrm{al}^{(17)}$ demonstrated better results for this outcome in the US-guided steroid injection group compared to the insole group.

\section{DISCUSSION}

All ten studies reviewed were consistent in showing that corticosteroid injections result in improvement of plantar fasciitis from baseline. The two high-quality placebo-controlled trials ${ }^{(13,14)}$ provided strong evidence of the effectiveness of corticosteroid injections in the reduction of both heel pain and plantar fascia thickness. This effect has been shown to last for up to three months in patients who had failed two months of conservative treatment.

\section{US- and palpation-guided corticosteroid injections}

The majority of studies investigated the use of palpation-guided corticosteroid injections, ${ }^{(7,15,16,18-21)}$ while two studies ${ }^{(14,17)}$ looked solely at US-guided corticosteroid injections. Only one study by Ball et al ${ }^{(13)}$ included both palpation- and US-guided corticosteroid injections for comparison against a placebo; however, no significant differences in heel pain reduction between the US- and palpation-guided corticosteroid injection groups were found. Similar results were seen in a recent meta-analysis (comprising five RCTs with 149 patients) conducted by Li et al, (22) in which heel pain measured with VAS was not shown to be significantly different between the US- and palpation-guided corticosteroid injection groups.

\section{Peppering technique}

This technique was first described in 1964 for lateral epicondylitis. When using this technique, the needle is 
Table II. Summary of physician-assessed and disease-oriented outcomes.

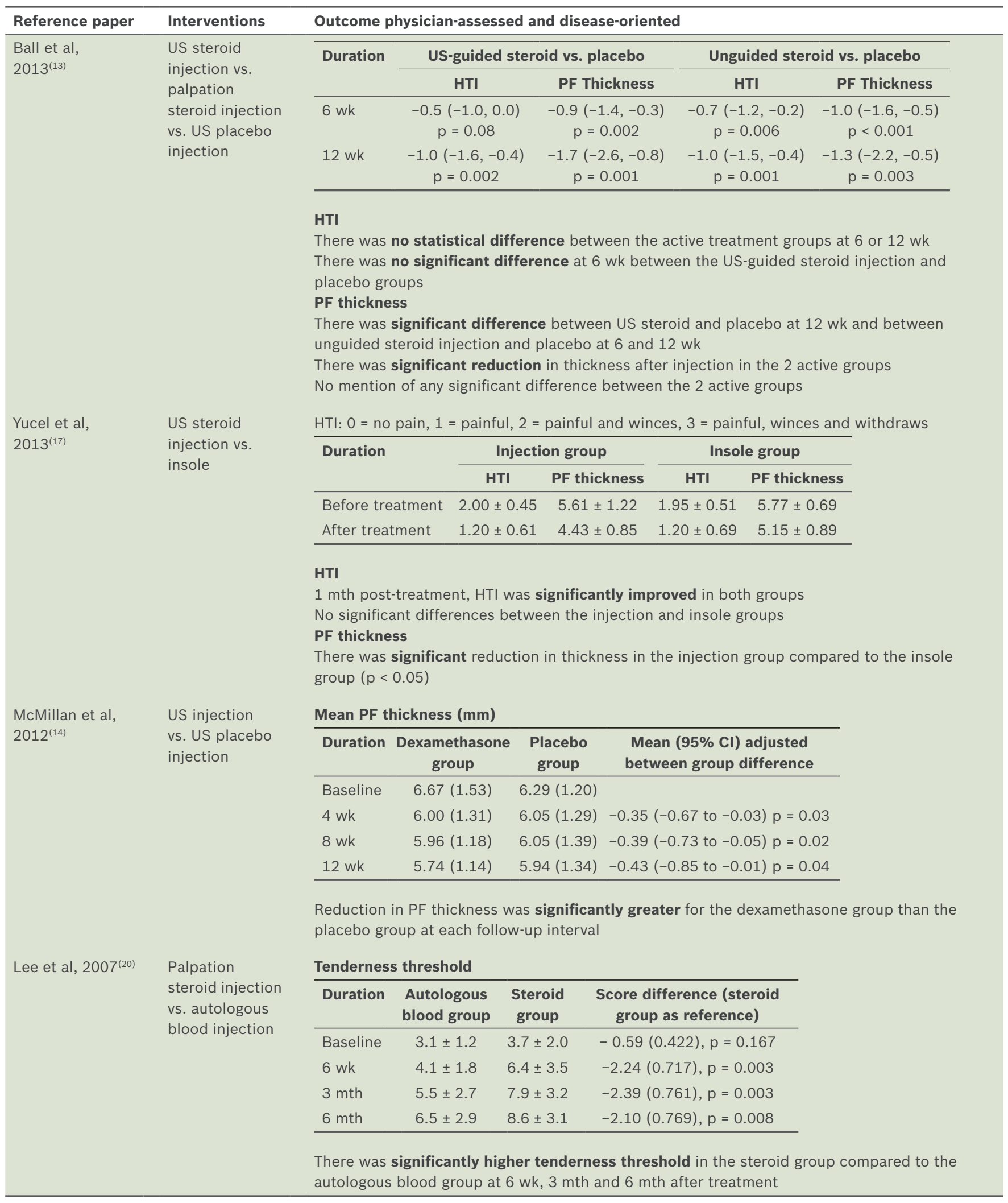

$\mathrm{Cl}$ : confidence interval; HTI: Heel Tenderness Index; PF: plantar fascia; US: ultrasonography

repeatedly inserted and withdrawn without complete emergence from the skin. It has been postulated that this repeated action leads to the creation of multiple small holes within the degenerative tissues, causing bleeding and initiating the healing process. In a three-arm study by Kiter et al,(21) this technique was compared with autologous blood and corticosteroid injections. All three groups were given prilocaine $1 \mathrm{~mL}$ prior to the administration of injections. The six-month assessment showed an improvement from baseline in all three groups $(65 \%-68 \%)$ but no significant differences between 
the groups. In a separate four-arm study by Kalaci et al, ${ }^{(23)}$ it was found that the peppering technique combined with corticosteroid injection resulted in a significantly lower VAS score for heel pain compared with corticosteroid injection alone. Kalaci et al's study was excluded from the present review, as it used consecutive patients instead of randomisation.

\section{Local anaesthesia and tibial nerve block}

Heel injections are regarded as painful. Thus, all the studies used either local or regional anaesthesia to mitigate the patients' pain. McMillan et al ${ }^{(14)}$ performed a US-guided posterior tibial nerve block prior to corticosteroid or placebo injections and found it effective in reducing the high level of pain experienced by patients during heel injections. Crawford et al's four-arm study, ${ }^{(7)}$ which examined the efficacy of corticosteroid injections, local anaesthesia and tibial nerve block, reported improvements in the mean pain scores of all the groups at the one-month followup compared to the baseline; however, the two corticosteroid injection groups in the study showed significantly better results compared to the non-steroid groups.

\section{Choice of corticosteroids}

The types of corticosteroids used for heel injections vary, as there is little evidence to suggest the superiority of one agent over the other. A meta-analysis by Gaujoux-Viala et al ${ }^{(24)}$ found no differences in efficacy between the various types of corticosteroids used. In the present review, all five types of corticosteroid injections used were found to result in significant heel pain reduction.

\section{Adverse effects}

Heel fat pad atrophy and plantar fascia rupture are two of the most feared complications associated with corticosteroid injections, as they can lead to intractable long-term complications. Various complication rates have been reported. The rupture rate of plantar fasciitis after corticosteroid injection ranged from $2.4 \%{ }^{(25)}$ to $6.7 \%{ }^{(26)}$ in two retrospective studies. The former study also found that patients with plantar fascia rupture received an average of 2.67 injections and had an average body mass index of $38.6 \mathrm{~kg} / \mathrm{m}^{2}$. A systematic review of RCTs and prospective studies by Brinks et $\mathrm{al}^{(27)}$ which examined the adverse effects of extraarticular corticosteroid injections, found only minor complications (i.e. post-injection heel pain) in 368 patients who were treated for plantar fasciitis and heel pain. This finding is largely similar to that of our review, which included 622 patients, as well as that of a meta-analysis of 149 patients conducted by Li et al. ${ }^{(22)}$

Three out of the ten $\operatorname{RCTs}^{(7,16,21)}$ reviewed in the present paper did not state any adverse outcomes of the corticosteroid injections, while the rest reported only post-injection heel pain. All but one of the RCTs had a follow-up period of six months or less. Hence, delayed complications such as plantar fascia rupture could have been under-reported. Although corticosteroid injection therapy in plantar fasciitis is generally associated with a low incidence of serious complications, multiple corticosteroid injections and obesity are potential risk factors for plantar fascia rupture.

\section{Comparison with other treatment modalities}

Two of the studies reviewed compared conservative therapies to corticosteroid injections. Ryan et $\mathrm{al}^{(16)}$ showed that participants who underwent seven physiotherapist-led exercises daily over a 12-week period had significant improvements during the sixweek and 12-week follow-up compared to baseline, although the improvement was not significantly better than the corticosteroid injection group. Yucel et al ${ }^{(17)}$ found that, at the one-month followup, the corticosteroid injection group reported significantly better pain relief than the group who wore a prefabricated full-length silicone insole daily for one month.

Three other injection modalities were used by five of the studies reviewed, namely tenoxicam, ${ }^{(15)}$ botulinum toxin $\mathrm{A}^{(18,19)}$ and autologous blood ${ }^{(20,21)}$ injections. Elizondo-Rodriguez et $\mathrm{al}^{(18)}$ showed that subjects who received botulinum toxin $A$ injections experienced significantly less heel pain at the six-month followup compared to those in the corticosteroid injection group. In contrast, Lee et $\mathrm{al}^{(20)}$ found that the corticosteroid group had significantly lower levels of heel pain six weeks and three months after treatment than the group that received autologous blood injection. The rest of the studies did not show any significant differences between the corticosteroid injection group and their comparator group.

\section{Limitations}

There are a number of limitations that should be considered when interpreting the results of this review. First, only two placebo-controlled RCTs were reviewed, while the rest of the RCTs compared only corticosteroid injections with other standard therapies. Generally, there are fewer available placebo-controlled trials, possibly due to ethical reasons, as patients who are in pain are exposed to a chance of non-intervention.

Second, half of the RCTs combined physical therapy or the peppering technique with corticosteroid injections as part of their intervention. ${ }^{(14-16,18,19)}$ This made it more difficult to interpret the magnitude of improvement resulting from corticosteroid injection alone. Furthermore, most of the studies had small sample sizes ranging from 40 to 106 participants. The types of corticosteroids used and the techniques of injection also varied, which added complexity to the interpretation of the results.

\section{RECOMMENDATIONS}

1. As the corticosteroid injections are associated with significant pain, local or regional anaesthesia should be used. However, there is currently no evidence to suggest that local or regional anaesthesia can bring about significant heel pain reduction in plantar fasciitis.

2. There is currently no indication for family physicians to change the injection technique from palpation-guided to US-guided, as the latter has not been shown to produce better results in our RCTs. In fact, patients in this arm experienced more pain during the procedure and typically required regional anaesthesia.

3. Although the peppering technique has been reported 
to be an effective technique in the treatment of lateral epicondylitis and tendinopathies in some studies, there is currently insufficient evidence to show that it is effective in treating plantar fasciitis.

4. There is currently no evidence to suggest the superiority of one type of corticosteroid over another. Therefore, the choice may depend on availability and the preference of the family physician.

5. There is some evidence to suggest that botulinum toxin A injections may produce better results than corticosteroid injections. However, further study is required to provide the necessary evidence.

6. Although the overall incidence of serious complications such as heel fat pad atrophy and plantar fascia rupture is low, they may be associated with long-term sequelae. Thus, physicians need to weigh the risks and benefits of the corticosteroid injection therapy for each patient.

7. There is a role for corticosteroid injections in patients with plantar fasciitis who still experience debilitating heel pain after unsuccessful conservative physical therapy. Non-obese patients who have not had prior corticosteroid injections are better candidates, as they are at a lower risk for plantar fascia rupture.

\section{CONCLUSION}

This review shows that both US- and palpation-guided corticosteroid injections are effective in reducing heel pain in patients with plantar fasciitis, including those with chronic pain and those who have failed conservative physical therapies. The effects are usually short term, lasting 4-12 weeks. The magnitude of pain reduction, as demonstrated by the placebo-controlled RCTs, ranges from $22.9 \%$ to $52.8 \%$. $^{(13,14)}$ No serious complications such as heel fat pad atrophy or plantar fascia rupture were reported by the studies reviewed in this paper. Although the incidence of such complications has been low in most studies, they may be associated with long-term sequelae. Thus, physicians need to weigh the risks and benefits of corticosteroid injection therapy for each patient.

\section{ACKNOWLEDGEMENTS}

I would like to thank the following for their help and guidance in making this research paper possible: Dr Tan Ngiap Chuan, Senior Consultant, SingHealth Polyclinics; and A/Prof Gerald Koh, Associate Professor, Saw Swee Hock School of Public Health and Joint Associate Professor, Dean's Office, Yong Loo Lin School of Medicine, National University of Singapore.

\section{REFERENCES}

1. Riddie DL, Schappert SM. Volume of ambulatory care visits and patterns of care for patients diagnosed with plantar fasciitis: a national survey of medical doctors. Foot Ankle Int 2004; 25:303-10.

2. Davis PF, Severud E, Baxter DE. Painful heel syndrome: results of nonoperative treatment. Foot Ankle Int 1994; 15:531-5.
3. Orchard J. Plantar fasciitis. BMJ 2012; 345:e6603.

4. Scher DL, Belmont PJ Jr, Bear R, et al. The incidence of plantar fasciitis in the United States military. J Bone Joint Surg Am 2009; 91:2867-72.

5. Schwartz En, Su J. Plantar fasciitis: a concise review. Perm J 2014; 18:e105-7.

6. Riddle DL, Pulisic M, Pidcoe P, Johnson RE. Risk factors for Plantar fasciitis: a matched case-control study. J Bone Joint Surg Am 2003; 85-A:872-7.

7. Crawford F, Atkins D, Young P, Edwards J. Steroid injection for heel pain: evidence of short-term effectiveness. A randomized controlled trial. Rheumatology (Oxford) 1999; 38:974-7.

8. Atkins D, Crawford F, Edwards J, Lambert M. A systematic review of treatments of the painful heel. Rheumatology(Oxford) 1999; 38:968-973

9. Lapidus PW, Guidotti FP. Local injections of hydrocortisone in 495 orthopedic patients. Ind Med Surg 1957; 26:234-44.

10. Fadale PD, Wiggins ME. Corticosteroid Injections: Their Use and Abuse. J Am Acad Orthop Surg 1994; 2:133-140.

11. Thomas JL, Christensen JC, Kravitz SR, et al; American College of Foot and Ankle Surgeons heel pain committee. The diagnosis and treatment of heel pain: a clinical practice guideline-revision 2010. J Foot Ankle Surg 2010; 49(3 Suppl): S1-19.

12. Jadad AR, Moore RA, Carroll D, et al. Assessing the quality of reports of randomized clinical trials: is blinding necessary? Control Clin Trials 1996; 17:1-12.

13. Ball EM, McKeeman HM, Patterson C, et al. Steroid injection for inferior heel pain: a randomised controlled trial. Ann Rheum Dis 2013; 72:9961002.

14. MCMillan AM, Landorf KB, Gilheany MF, et al. Ultrasound guided corticosteroid injection for plantar fasciitis: randomised controlled trial. BMJ 2012; 344:e3260

15. Guner S, Onder H, Guner SI, et al. Effectiveness of local tenoxicam versus corticosteroid injection for plantar fasciitis treatment. Orthopedics 2013; 36:e1322-6.

16. Ryan M, Hartwell J, Fraser S, Newsham-West R, Taunton J. Comparison of a physiotherapy program versus dexamethasone injections for plantar fasciopathy in prolonged standing workers: a randomized clinical trial. Clin J Sport Med 2014; 24:211-7.

17. Yucel U, Kucuksen S, Cingoz HT, et al. Full-length silicone insoles versus ultrasound-guided corticosteroid injection in the management of plantar fasciitis: a randomized clinical trial. Prosthet Orthot Int 2013; 37:471-6.

18. Elizondo-Rodriguez J, Araujo-Lopez Y, Moreno-Gonzalez JA, et al. A comparison of botulinum toxin a and intralesional steroids for the treatment of plantar fasciitis: a randomized, double-blinded study. Foot Ankle Int 2013; 34:8-14.

19. Díaz-Llopis IV, Rodríguez-Ruíz CM, Mulet-Perry S, et al. Randomized controlled study of the efficacy of the injection of botulinum toxin type A versus corticosteroids in chronic plantar fasciitis: results at one and six months. Clin Rehabil 2012; 26:594-606.

20. Lee TG, Ahmad TS. Intralesional autologous blood injection compared to corticosteroid injection for treatment of chronic plantar fasciitis. A prospective, randomized, controlled trial. Foot Ankle Int 2007; 28:984-90.

21. Kiter E, Celikbas E, Akkaya S, Demirkan F, Kiliç BA. Comparison of injection modalities in the treatment of plantar heel pain: a randomized controlled trial. J Am Podiatr Med Assoc 2006; 96:293-6.

22. Li Z, Xia C, Yu A, Qi B. Ultrasound- versus palpation-guided injection of corticosteroid for plantar fasciitis: a meta-analysis. PLoS One 2014; 9:e92671.

23. Kalaci A, Cakici $\mathrm{H}$, Hapa O, et al. Treatment of plantar fasciitis using four different local injection modalities: a randomized prospective clinical trial. J Am Podiatr Med Assoc 2009; 99:108-13.

24. Gaujoux-Viala C, Dougados M, Gossec L. Efficacy and safety of steroid injections for shoulder and elbow tendonitis: a meta-analysis of randomised controlled trials. Ann Rheum Dis 2009; 68:1843-9.

25. Kim C, Cashdollar MR, Mendicino RW, Catanzariti AR, Fuge L. Incidence of plantar fascia ruptures following corticosteroid injection. Foot Ankle Spec 2010; 3:335-7.

26. Acevedo JI, Beskin JL. Complications of plantar fascia rupture associated with corticosteroid injection. Foot Ankle Int 1998; 19:91-7.

27. Brinks A, Koes BW, Volkers AC, Verhaar JA, Bierma-Zeinstra SM. Adverse effects of extra-articular corticosteroid injections: a systematic review. BMC Musculoskelet Disord 2010; 11:206. 